$\begin{gathered}\text { Revista do Departamento de Geografia } \\ \text { Universidade de São Paulo } \\ \text { www.revistas.usp.br/rdg }\end{gathered}$
Volume Especial - XVII SBGFA / I CNGF (2017)
ISSN 2236-2878

\title{
Modelos de Erosão Hídrica e Tolerância das Perdas de Solo em Latossolos Distróficos no Sul de Minas Gerais
}

\section{Models of Water Erosion and Soil Loss Tolerance in Dystrophic Oxisols in Southern Minas Gerais}

\author{
André Silva Tavares \\ Universidade Federal de Alfenas \\ andresttavares@gmail.com \\ Henrique Mendes Júnior \\ Universidade Federal de Alfenas \\ mendesjr49@gmail.com \\ Velibor Spalevic \\ University of Montenegro \\ velibor.spalevic@gmail.com \\ Ronaldo Luiz Mincato \\ Universidade Federal de Alfenas \\ ronaldo.mincato@unifal-mg.edu.br
}

\begin{abstract}
Resumo: Diferentes métodos de cálculo das perdas de solo por erosão hídrica foram comparados com a Tolerância de Perda de Solo em Latossolos tropicais. Foram utilizados o Método de Erosão Potencial e a Equação Universal de Perda de Solo Revisada. O Método de Erosão Potencial foi calculado no aplicativo Intensidade de Erosão e Escoamento e obteve uma média de perdas de solo de 1,46 $\mathrm{Mg} \mathrm{ha}{ }^{-1}$ ano $^{-1}$ com descarga máxima de 649,31 Mg ano ${ }^{-1}$. Já pela Equação Universal de Perda de Solo Revisada, foi obtida uma média de perda de solo de $1,52 \mathrm{Mg} \mathrm{ha}^{-1} \mathrm{ano}^{-1}$, com descarga máxima de $648,87 \mathrm{Mg} \mathrm{ano}^{-1}$. As perdas de solo calculadas pelos dois métodos foram similares e ficaram aquém do limite de Tolerância de Perda de Solo, que variou de 5,19 a 5,90 $\mathrm{Mg} \mathrm{ha}^{-1}$ ano $^{-1}$ e validam a aplicação do Método da Erosão Potencial em solos tropicais do sul de Minas Gerais.
\end{abstract}

Palavras-chave: EPM; RUSLE; IntErO; Degradação do Solo.

\begin{abstract}
Different methods of calculating soil losses by water erosion were compared with Soil Loss Tolerance in tropical Oxisols. The Potential Erosion Method and the Revised Universal Soil Loss Equation were used. The Potential Erosion Method was calculated by the Erosion and Flow Intensity software and an average soil loss of $1.46 \mathrm{Mg} \mathrm{ha}^{-1}$ year ${ }^{-1}$ with a maximum discharge of 649.31 Mg year ${ }^{-1}$ was obtained. Alternatively, an average soil loss of $1.52 \mathrm{Mg} \mathrm{ha}^{-1}$ year-1 with a maximum discharge of 668.26 Mg year-1 was obtained by the Universal Equation of Revised Soil Loss. Soil losses calculated by the two methods were similar and fell below the Soil Loss Tolerance limit, which ranged from 5.19 to $5.90 \mathrm{Mg} \mathrm{ha}^{-1}$ year ${ }^{-1}$ and validate the application of the Potential Erosion Method in Tropical soils of southern Minas Gerais State.
\end{abstract}

Keywords: EPM; RUSLE; IntErO; Soil Degradation. 


\section{INTRODUÇÃO}

O processo de aceleração da erosão hídrica por ações antrópicas causa perdas de solo, água e nutrientes essenciais para o equilíbrio sustentável da agropecuária (PANAGOS et al., 2015). As taxas de perdas de solo no Brasil variam de 15 a $25 \mathrm{Mg} \mathrm{ha}^{-1}$ ano $^{-1}$, enquanto que as de formação do solo estão em cerca de $1 \mathrm{Mg}$ ha-1 ano-1 (BERTONI E LOMBARDI NETO, 2012; PIMENTEL et al., 1995).

Métodos empíricos como a Revised Universal Soil Loss Equation, RUSLE (RENARD et al., 1997) e físicos como o Erosion Potential Method, EPM (GAVRILOVIC, 1988), foram desenvolvidos para modelar as perdas de solo por erosão hídrica a partir de técnicas de geoprocessamento, imagens de satélites e atributos físicos e químicos do solo.

A modelagem das perdas de solo permite o conhecimento das áreas com maior intensidade de erosão e a comparação com o limite da Tolerância de Perda de Solo (TPS). A TPS representa a perda de solo máxima tolerável para manter a capacidade produtiva do solo e no Brasil é calculada, principalmente, pelo método de Bertol e Almeida (2000), por considerar no cálculo um maior número de atributos do solo de fácil obtenção (CÂNDIDO et al., 2014; OLIVETTI et al., 2015).

A região do sul de Minas Gerais possui grandes áreas de cultivo do café, porém com carência de avaliações das perdas de solo. Carvalho et al. (2007) calcularam as perdas de solo em Latossolos da região com parcelas padrões pelo método da Equação Universal de Perdas de Solos (Universal Soil Loss Equation USLE) (WISCHMEIER E SMITH, 1978) e verificaram perdas de solos menores nos sistemas de manejo mais adensados em comparação aos mais espaçados. Portanto, a modelagem da erosão hídrica permite quantificar as perdas de solo e comparar à TPS, visando avaliar e orientar as práticas de manejo necessárias para a sustentabilidade no uso do solo.

Assim, neste trabalho foram calculadas as perdas de solo por erosão hídrica pelos modelos RUSLE e EPM e comparadas com os limites da TPS em Latossolos sob diferentes usos.

\section{MATERIAIS E MÉTODOS}

A área de estudo compreende a Sub-bacia Hidrográfica do Córrego da Laje, com área de 437 ha, situada na Fazenda Capoeirinha da Ipanema Agrícola S.A. (Ipanema Coffees), nas coordenadas UTM 402000 a 405000 m E e 7616700 a 7620200 m N, zona 23K, Datum SIRGAS 2000 (Figura 1). A Sub-bacia do Córrego da Laje possui perímetro de 9,90 km e altitudes entre 814 e $914 \mathrm{~m}$ e é afluente direta do Reservatório da Usina Hidrelétrica de Furnas, na Bacia Hidrográfica do Rio Grande, sul de Minas Gerais.

O clima, na classificação de Köppen, é o Tropical Mesotérmico (CwB), com precipitação média de $1.500 \mathrm{~mm} \mathrm{ano}^{-1}$ (SPAROVEK et al., 2007). O relevo pertence à unidade fisiográfica dos mares de morros, na formação da Unidade litológica Paragnáissica Migmatítica Superior Varginha - Guaxupé (HASUI, 2010).

As classes de uso do solo da sub-bacia são: café (237,35 ha); milho e feijão com cultivo em sucessão (60,49 ha); eucalipto (16,95 ha); área de preservação permanente (APP) $(90,59$ ha); carreadores (vias de acesso) (9,71 ha); usina de adubo (2,40 ha) e drenagens (19,51 ha). As APP equivalem a 20,73\% da sub-bacia e estão de acordo com o Código Florestal Brasileiro (BRASIL, 2012) respeitando os $50 \mathrm{~m}$ para o entorno das nascentes e os $30 \mathrm{~m}$ para margens dos cursos d'água. A área de mata nativa considerada nos cálculos das perdas de solo foi de 68,99 ha.

O Modelo Digital de Elevação (MDE), com resolução (pixels) de 10 m (Figura 1), foi obtido a partir da interpolação das curvas de nível da Carta Topográfica de Alfenas (FOLHA SF 23-1-1-3) na escala de 1: 50.000 (IBGE, 1970) pela ferramenta topo to raster do software ArcGis 10.3. 


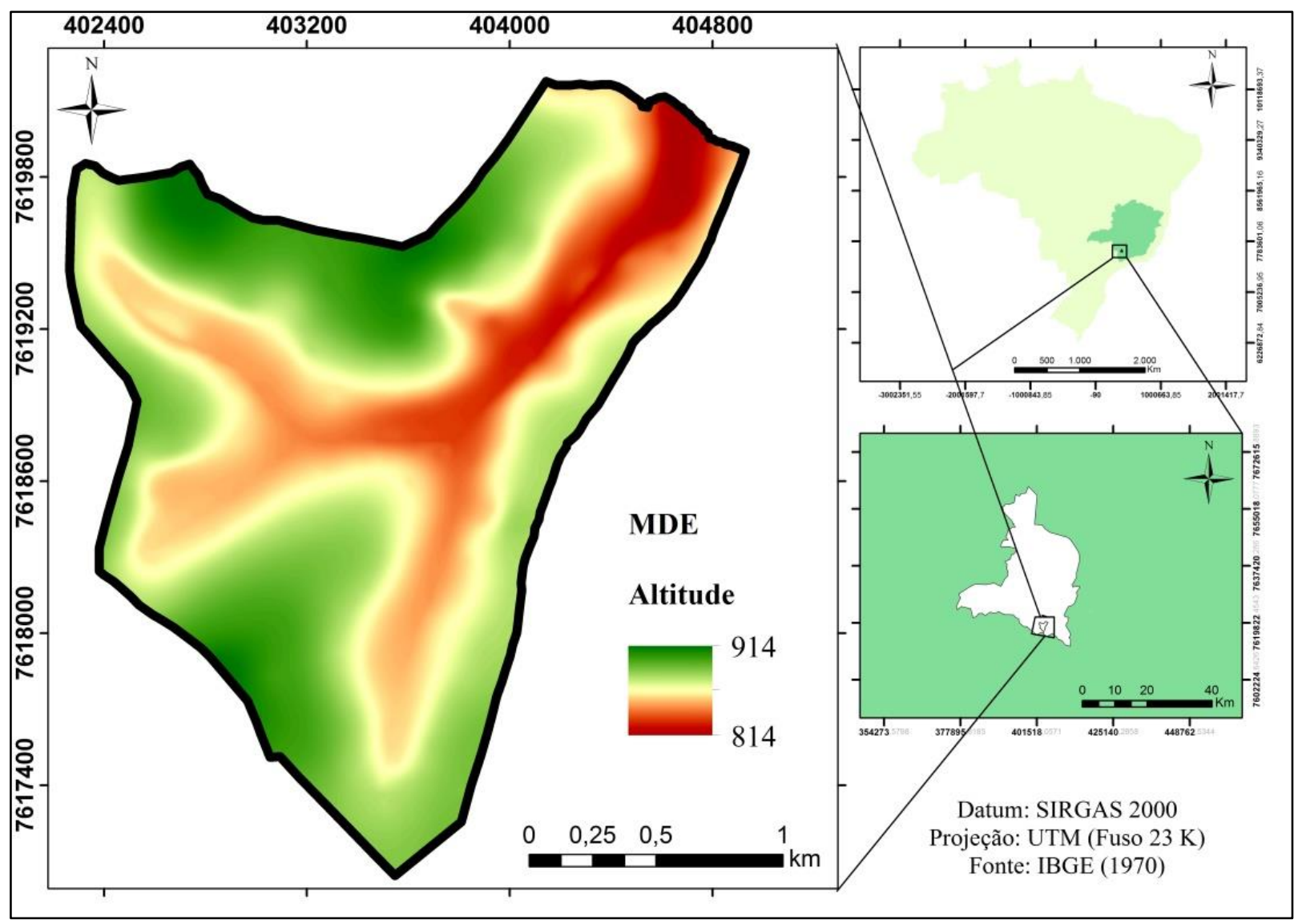

Figura 1 - Mapa de localização e Modelo Digital de Elevação da Sub-bacia Hidrográfica do Córrego da Laje, Alfenas, sul de Minas Gerais.

A partir do MDE foi confeccionado o mapa de declividade, por meio da ferramenta slope do ArcGis 10.3, que serviu como base para a obtenção das classes de solo, conforme o modelo Scorpan de McBratney et al. (2003). O mapa digital de solos foi elaborado com base nas características do solo. Para tanto foram coletadas 108 amostras: entre deformadas, indeformadas em cilindro $\left(92,53 \mathrm{~cm}^{3}\right)$ e indeformadas em forma de torrão.

As análises de solo foram realizadas nos laboratórios do Departamento de Ciência do Solo da Universidade Federal de Lavras - UFLA. A descrição morfológica foi realizada no campo em micro trincheiras (40 x 40 x $60 \mathrm{~cm}$ ), conforme os procedimentos de Santos et al. (2005). O mapa digital de solos em nível semidetalhado (IBGE, 2015) considerou as classes de relevo da Embrapa (2013) (Figura 2). Os solos foram classificados em Latossolo Vermelho distrófico em relevo plano a suave ondulado (LVd1), ondulado (LVd2), forte ondulado (LVd3) e solos indiscriminados de várzea (SIV) nas zonas de deposição. 


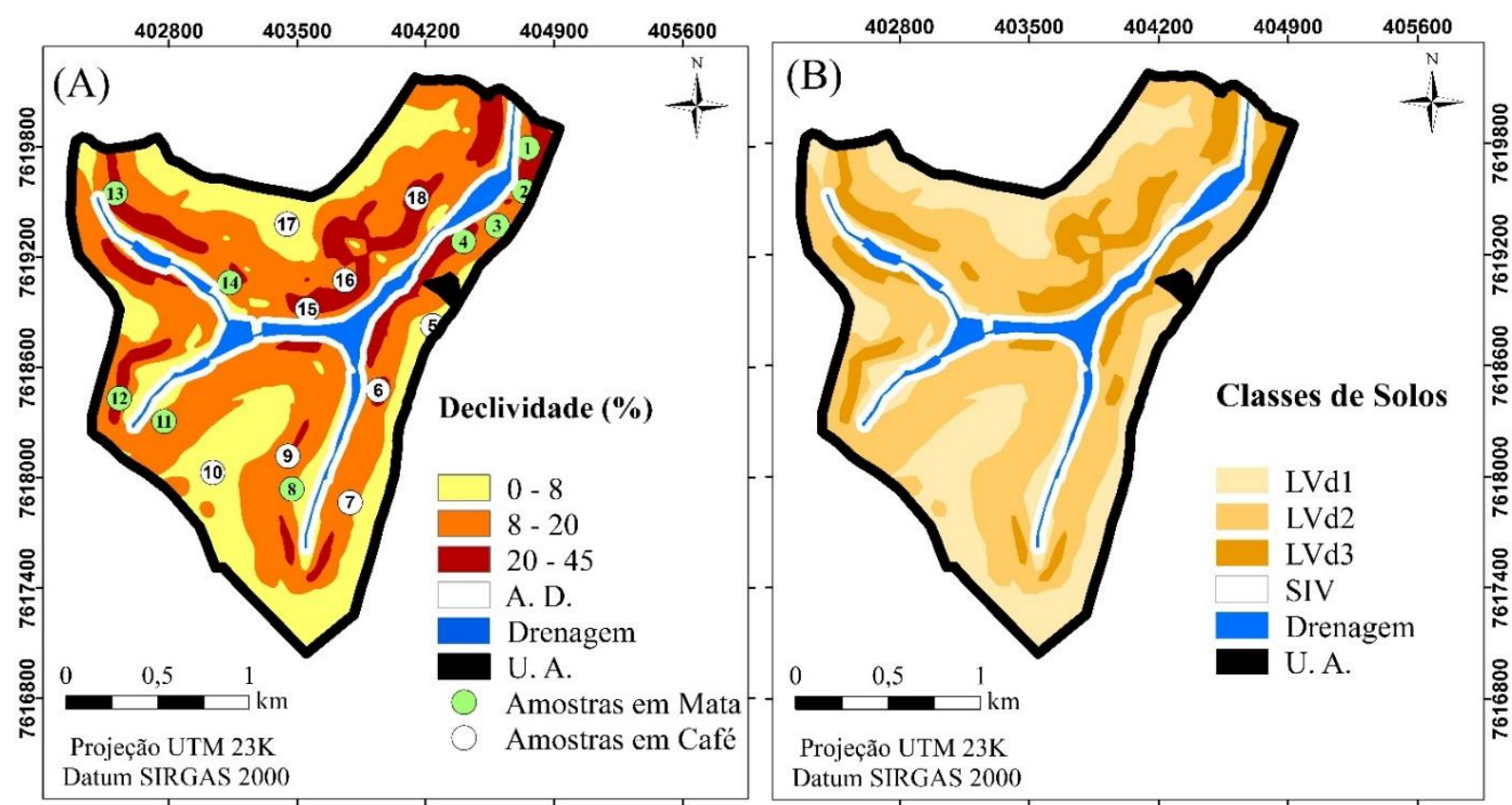

Figura 2: (A) Mapa de declividade e (B) Mapa digital de solos da Sub-bacia hidrográfica do Córrego da Laje, Alfenas, sul de Minas Gerais. Latossolo Vermelho Distrófico em relevo plano a suave ondulado (LVd1), ondulado (LVd2) e forte ondulado (LVd3). Solos Indiscriminados de Várzea (SIV).

\section{Perdas de Solo no Modelo Rusle}

A perda média de solo pelo modelo RUSLE (RENARD et al., 1997) é baseada na Equação 1.

$$
\mathrm{A}=\mathrm{R} \cdot \mathrm{K} \cdot \mathrm{LS} \cdot \mathrm{C} \cdot \mathrm{P} \quad(\text { Equação 1) }
$$

Em que: $\mathrm{A}=$ perda de solo média anual $\left(\mathrm{Mg} \mathrm{ha}^{-1} \mathrm{ano}^{-1}\right) ; \mathrm{R}=$ erosividade da chuva $\left(\mathrm{MJ} \mathrm{mm} \mathrm{ha-1} \mathrm{h}^{-1}\right.$ ano $\left.{ }^{-1}\right) ; \mathrm{K}$ $=$ erodibilidade do solo $\left(\mathrm{Mg} \mathrm{h} \mathrm{MJ} \mathrm{mm}^{-1}\right) ; \mathrm{LS}=$ fator topográfico, que envolve o comprimento e a declividade da rampa (adimensional); $\mathrm{C}=$ uso e manejo do solo (adimensional); $\mathrm{P}=$ práticas conservacionistas no solo (adimensional).

A erosividade da chuva (R) foi obtida de Aquino et al. (2012). A erodibilidade do solo (K) foi calculada com base nas descrições morfológicas e análises físicas das amostras coletadas sob mata nativa, utilizando o método indireto número 1 de Silva et al. (1999), expresso na Equação 2.

$\mathrm{Y}=4,77 \cdot 10^{-2}-9,66 \cdot 10^{-3} \cdot X_{14}+1,63 \cdot 10^{-2} \cdot X_{16}-1,12 \cdot 10^{-2} \cdot X_{17}+1,85 \cdot 10^{-2} \cdot X_{18}-1,51$. $10^{-2} \cdot X_{19}-2,46 \cdot 10^{-4} \cdot X_{22}-3,58 \cdot 10^{-4} \cdot X_{23}+1,47 \cdot 10^{-4} \cdot X_{24}-1,43 \cdot 10^{-4} \cdot X_{25}+3,26$. $10^{-3} \cdot X_{26}-1,26 \cdot 10^{-3} \cdot X_{27}-2,29 \cdot 10^{-4} \cdot X_{31}+1,07 \cdot 10^{-4} \cdot X_{32}+2,69 \cdot 10^{-4} \cdot X_{34}$

(Equação 2)

Em que: $\mathrm{Y}=$ erodibilidade em $\mathrm{Mg} \mathrm{h} \mathrm{MJ}^{-1} \mathrm{~mm}^{-1} ; \mathrm{X}_{14}=$ código do matiz do solo úmido segundo Munsell (adimensional); $\mathrm{X}_{16}=$ código do grau da estrutura (adimensional); $\mathrm{X}_{17}=$ código do tamanho da estrutura (adimensional); $\mathrm{X}_{18}=$ código da forma da estrutura (adimensional); $\mathrm{X}_{19}=$ código da plasticidade do solo (adimensional); $\mathrm{X}_{22}=$ teor de areia fina dispersa com NaOH $0,1 \mathrm{~mol} \mathrm{~L}^{-1}\left(\mathrm{~g} \mathrm{~kg}^{-1}\right) ; \mathrm{X}_{23}=$ teor de areia muito fina dispersa com NaOH 0,1 mol L-1 $\left(\mathrm{g} \mathrm{kg}^{-1}\right) ; \mathrm{X}_{24}=$ teor de silte disperso com NaOH 0,1 mol L-1 $\left(\mathrm{g} \mathrm{kg}^{-1}\right) ; \mathrm{X}_{25}$ $=$ teor de argila dispersa com $\mathrm{NaOH} 0,1 \mathrm{~mol} \mathrm{~L}^{-1}\left(\mathrm{~g} \mathrm{~kg}^{-1}\right) ; \mathrm{X}_{26}=$ teor de areia muito grossa disperso em água $\left(\mathrm{g} \mathrm{kg}^{-1}\right) ; \mathrm{X}_{27}=$ teor de areia grossa disperso em água $\left(\mathrm{g} \mathrm{kg}^{-1}\right) ; \mathrm{X}_{31}=$ teor de silte disperso em água $\left(\mathrm{g} \mathrm{kg}^{-1}\right)$; $\mathrm{X}_{32}=$ teor de argila dispersa em água $\left(\mathrm{g} \mathrm{kg}^{-1}\right)$ e $\mathrm{X}_{34}=$ índice de floculação (adimensional). 
As variáveis $\mathrm{X}_{14}, \mathrm{X}_{16}, \mathrm{X}_{17}, \mathrm{X}_{18}$ e $\mathrm{X}_{19}$ foram obtidas pela descrição morfológica do solo em campo. As variáveis $\mathrm{X}_{22}, \mathrm{X}_{23}, \mathrm{X}_{24}, \mathrm{X}_{25}, \mathrm{X}_{26}, \mathrm{X}_{27}, \mathrm{X}_{31} \mathrm{e} \mathrm{X}_{32}$ foram determinadas em laboratório pela análise granulométrica do solo. A variável $X_{34}$ foi obtida de Embrapa (2011). Equação 3.

A topografia (LS) foi determinada pelo modelo $\mathrm{LS}_{\mathrm{RUSLE}}$ 3D de Mitasova et al. (1996), representado pela

$$
\mathrm{LS}_{\text {RUSLE 3D }}=(\mathrm{m}+1) \cdot\left(\frac{\mathrm{A}}{22,13}\right)^{m} \cdot\left(\frac{\operatorname{sen} \theta}{0,09}\right)^{n}
$$

Em que: $\mathrm{LS}_{\mathrm{RUSLE}} 3 \mathrm{D}=$ fator topográfico (adimensional); $\mathrm{A}=$ área de contribuição a montante por unidade de comprimento de célula para um $\operatorname{MDE}\left(\mathrm{m}^{2}\right) ; \Theta=$ ângulo de inclinação do declive (graus) e $\mathrm{m}$ e $\mathrm{n}=$ parâmetros empíricos com variação do $m$ entre 0,4 a 0,6 e do $n$ entre 1,0 a 1,4 em função do tipo de erosão predominante (laminar ou em sulcos).

Os fatores de uso e manejo do solo $(\mathrm{C})$ e práticas conservacionistas $(\mathrm{P})$ foram obtidos da literatura especializada para cada uso do solo e do mapa das classes de uso do solo.

$\mathrm{O}$ fator $\mathrm{P}$ foi determinado com base nas práticas conservacionistas adotadas na área e o mapa das classes de uso do solo. O cultivo do café é realizado em curvas de nível com plantio em contorno e o milho e o feijão com plantio direto. Nos povoamentos de eucalipto não foi verificada nenhuma prática conservacionista e foi caracterizado como plantio morro abaixo. Para mata nativa foi atribuído valor zero por ser área de preservação.

\section{Perdas de Solo no Modelo EPM}

A perda de solo (Wyr) no modelo EPM é estimada pela Equação 4 (GAVRILOVIC, 1988). Os seus algoritmos estão incorporados no aplicativo IntErO (SPALEVIC, 2011), que calcula de forma automatizada, evitando os erros na modelagem manual.

$$
\text { Wyr }=\mathrm{T} \cdot \mathrm{Hyr} \cdot \pi \cdot \sqrt[2]{Z^{3}} \cdot \mathrm{F} \quad(\text { Equação 4) }
$$

Em que: $\mathrm{Wyr}=$ produção total de sedimentos $\left(\mathrm{m}^{3}\right.$ ano $\left.^{-1}\right) ; \mathrm{T}=$ coeficiente de temperatura (adimensional); Hyr $=$ precipitação média $\left(\mathrm{mm}\right.$ ano $\left.^{-1}\right) ; \pi=3,14 ; \mathrm{Z}=$ coeficientes de erosão (adimensional); $\mathrm{F}=$ área da bacia hidrográfica $\left(\mathrm{km}^{2}\right)$.

O coeficiente de temperatura $(\mathrm{T})$ é calculado conforme a Equação 5.

$$
\mathrm{T}=\sqrt[2]{\left(\frac{t 0}{10}\right)}+0,1
$$

Em que: $\mathrm{T}=$ coeficiente de temperatura (adimensional); $\mathrm{t} 0=$ temperatura média do ar $\left({ }^{\circ} \mathrm{C}\right.$ ano $\left.{ }^{-1}\right)$.

A temperatura média do ar ( $\mathrm{t} 0$ ) é de $22^{\circ} \mathrm{C}$, com máximas variando de 28 a $30^{\circ} \mathrm{C}$ nos períodos mais secos e no inverno as médias mensais variam 17 a $19^{\circ} \mathrm{C}$.

O coeficiente de erosão (Z) é obtido pela Equação 6:

$$
\mathrm{Z}=\mathrm{Y} \cdot \mathrm{Xa} \cdot(\varphi+\mathrm{Isr})
$$

(Equação 6)

Em que: $\mathrm{Y}=$ resistência do solo a erosão hídrica (adimensional); $\mathrm{Xa}=$ uso e manejo do solo (adimensional); $\varphi=$ grau de erosão no terreno (adimensional); Isr = inclinação média da sub-bacia (\%). 
Gavrilovic (1988) preparou tabelas com valores que representam os atributos (Y, Xa, $\varphi$ ) necessários para o cálculo do coeficiente de erosão (Z). Entretanto, o modelo foi aplicado inicialmente em regiões de clima temperado, sendo necessário adaptar os valores de acordo com as características dos solos tropicais brasileiros.

O coeficiente de retenção de sedimentos $(\mathrm{Ru})$ é calculado pela Equação 7.

$$
\mathrm{Ru}=\frac{(\mathrm{O} \cdot \mathrm{D})^{0,5}}{0,25 \cdot(\mathrm{Lv}+10,0)}
$$

(Equação 7)

Em que: $\mathrm{O}=$ comprimento da sub-bacia $(\mathrm{km}) ; \mathrm{D}=$ diferença de elevação da sub-bacia $(\mathrm{m}) ; \mathrm{Lv}=$ comprimento do córrego principal da sub-bacia $(\mathrm{km})$.

\section{Tolerância de Perdas de Solo}

A TPS foi obtida pelo método de Bertol e Almeida (2000) (Equação 8).

$$
\text { TPS }=\mathrm{h} \cdot \mathrm{r}_{a} \cdot \mathrm{m} \cdot \mathrm{p} \cdot 1.000^{-1} \quad(\text { Equação 8) }
$$

Em que: TPS = tolerância de perdas de solo $\left(\mathrm{em} \mathrm{Mg} \mathrm{ha}^{-1}\right.$ ano $\left.^{-1}\right)$, corrigida da fórmula original considerando a densidade do solo; $h=$ profundidade efetiva do solo $(\mathrm{mm})$, limitada a $1.000 \mathrm{~mm} ; \mathrm{r}_{\mathrm{a}}=$ relação que expressa, conjuntamente, o efeito da relação textural entre os horizontes $\mathrm{B}$ e A e do teor de argila do horizonte $\mathrm{A} ; \mathrm{m}=$ fator que expressa o efeito da matéria orgânica na camada de $0-20 \mathrm{~cm}$ do solo; $p=$ fator que expressa o efeito da permeabilidade do solo e $1.000^{-1}=$ constante que expressa o período de tempo necessário para desgastar uma camada de solo de $1.000 \mathrm{~mm}$ de espessura.

A profundidade efetiva (variável h) para todas as unidades de mapeamento foi de $1.000 \mathrm{~mm}$ (BRASIL, 1962). Para a variável $r_{a}$ foi utilizado o teor de argila dispersa com $\mathrm{NaOH} 0,1 \mathrm{~mol} \mathrm{~L}^{-1}$ que variou de 584 a 607 $\mathrm{g} \mathrm{kg}^{-1}$. Assim, primeiro, foi obtido a relação textural entre as camadas superficial $(0-20 \mathrm{~cm})$ e subsuperficial $(20-60 \mathrm{~cm})$ de cada amostragem (EMBRAPA, 2013). A variável $\mathrm{m}$ representa o teor médio de matéria orgânica na camada superficial $(0-20 \mathrm{~cm})$ de cada classe de solo. A variável permeabilidade do solo, $\mathrm{p}$, foi obtida em campo, a partir de três repetições para cada classe de solo (ZHANG, 1997) com Infiltrômetro Mini Disk Decagon Devices ajustado para taxa de sucção de $2 \mathrm{~cm}$. A permeabilidade do solo foi classificação de acordo com Soil Survey Division Staff (1993) e Galindo e Margolis (1989) que utilizam a textura e o grau de estrutura do solo.

\section{RESULTADOS E DISCUSSÕES}

\section{Modelo Rusle}

$\mathrm{O}$ fator R para a área é de $6.500 \mathrm{MJ} \mathrm{mm} \mathrm{ha} \mathrm{m}^{-1} \mathrm{~h}^{-1} \mathrm{ano}^{-1}$ (AQUINO et al., 2012). O fator $\mathrm{K}$ foi de 0,021 , 0,004 e $0,026 \mathrm{Mg} \mathrm{h} \mathrm{MJ}^{-1} \mathrm{~mm}^{-1}$, respectivamente, para LVd1, LVd2 e LVd3. O fator LS variou de 0 a 3,883, com média de 0,318 . Os valores da literatura utilizados para os fatores $\mathrm{C}$ e $\mathrm{P}$ estão listados na Tabela 1.

Tabela 1: Fatores C e P para os usos e manejos verificados na Sub-bacia Hidrográfica do Córrego da Laje.

\begin{tabular}{l|c|c|c}
\hline \multicolumn{1}{c|}{ Uso e Manejo } & $\begin{array}{c}\text { Fator } \\
\mathbf{C}\end{array}$ & $\begin{array}{c}\text { Fator } \\
\mathbf{P} \mathbf{1}\end{array}$ & Autor \\
\hline Café com espaçamento de 3,0 x 0,5 m e plantio em nível & 0,1354 & 0,50 & Prochnow et al. (2005) \\
\hline Milho + feijão com plantio direto & 0,0271 & 0,01 & Bertol et al. $(2001)$ \\
\hline Eucalipto & 0,1240 & 1,00 & Silva et al. $(2016)$ \\
\hline Mata nativa & 0,0150 & 0,00 & Silva et al. (2016) \\
\hline Carreadores (vias de acesso) & 1,0000 & 1,00 & \\
\hline
\end{tabular}

Nota: ${ }^{1}$ Valores de P obtidos de Bertoni e Lombardi Neto (2012) e Roose (1977). 
A perda de solo média foi de $1,52 \mathrm{Mg} \mathrm{ha}^{-1}$ ano $^{-1}$ com variação de 0,05 a $18,64 \mathrm{Mg} \mathrm{ha}^{-1}$ ano $^{-1}$. Os maiores valores estão concentrados nos carreadores do café, com 18,64 $\mathrm{Mg} \mathrm{ha}^{-1} \mathrm{ano}^{-1}$, que também possui os fatores C, $\mathrm{P}$ e LS elevados pelo fato de configurarem solos expostos posicionados, na sua maioria, em terreno de declividade forte ondulada $(20-45 \%)$. Já os menores valores de perda do solo estão associados à mata nativa com $0,05 \mathrm{Mg} \mathrm{ha}^{-1}$ ano $^{-1}$. Os cultivos do milho e feijão em sucessão apresentaram perdas de solo médias de 0,04 $\mathrm{Mg} \mathrm{ha}^{-1}$ ano $^{-1}$, o eucalipto de 6,05 $\mathrm{Mg} \mathrm{ha}^{-1}$ ano $^{-1}$ e o café de $1,52 \mathrm{Mg} \mathrm{ha}^{-1}$ ano $^{-1}$ (Tabela 2). Este valor para o café foi abaixo do encontrado por Silva et al. (2007), de 10,98 $\mathrm{Mg} \mathrm{ha}^{-1} \mathrm{ano}^{-1}$ para Latossolo Vermelho Amarelo Distrófico com café conilon em espaçamento 2,90 x 0,90 m cultivado há cinco anos em Cachoeiro de Itapemirim - ES. Essa diferença pode estar associada à distinção entre a erodibilidade dos solos, de 0,14 $\mathrm{Mg} \mathrm{h} \mathrm{MJ}^{-1} \mathrm{~mm}^{-1}$ (SILVA et al., 2007) e de 0,004 a $0,026 \mathrm{Mg} \mathrm{h} \mathrm{MJ}^{-1} \mathrm{~mm}^{-1}$ neste trabalho.

\section{Modelo EPM}

A precipitação média (Hyr) é de $1.500 \mathrm{~mm}^{2}$ ano $^{-1}$ (SPAROVEK et al., 2007). O coeficiente de temperatura (T) obtido foi de 1,52. A diferença de elevação na sub-bacia (D), que representa a distância média percorrida pelo escoamento até o deflúvio, foi de $37,21 \mathrm{~m}$. O perímetro (O) é de $9,90 \mathrm{~km}$ e o comprimento do córrego principal (Lv) é de 2,98 km, com largura média de $40 \mathrm{~m}$.

De acordo com o mapa de declividade, a inclinação media da sub-bacia (Isr) foi de 11,6\%, o que indica um relevo com declividade média. A resistência à erosão hídrica $(\mathrm{Y})$ dos Latossolos Vermelhos distróficos foi calculada em 0,90 , na escala de 0,25 a 2,0, que indica uma resistência moderada à erosão hídrica. A média do coeficiente para uso e manejo $(\mathrm{Xa})$ foi de 0,55 , o que indica um bom manejo no uso do solo, isso devido às práticas de conservação nos plantios em nível e direto, em mais de $60 \%$ da área.

O valor médio para o grau de erosão no terreno $(\varphi)$ foi de 0,40 , o que sugere processos de erosão do tipo fraco considerando a área da sub-bacia. A intensidade média da erosão $(Z)$ foi de 0,37 , indicando que a sub-bacia é classificada na categoria IV de deterioração, ou seja, de erosão do tipo fraca. O coeficiente de retenção de sedimentos $(\mathrm{Ru})$ foi de 0,093 , ou seja, $9,3 \%$ dos sedimentos produzidos no total das perdas de solo são transportados ao deflúvio.

A perda média de solo foi de $1,46 \mathrm{Mg} \mathrm{ha}^{-1} \mathrm{ano}^{-1}$, variável de 0,10 a 5,50 $\mathrm{Mg} \mathrm{ha}^{-1} \mathrm{ano}^{-1}$. As áreas com maior perda estão concentradas nos carreadores de café, com 5,50 $\mathrm{Mg} \mathrm{ha}^{-1} \mathrm{ano}^{-1}$. Os maiores valores são devidos ao solo exposto nos terrenos com declividades forte onduladas $(20-45 \%)$. Essas áreas são prioritárias para aplicação de práticas de conservação do solo e da água, visto que o uso intensivo de maquinários de grande porte contribui para a compactação e impermeabilização do solo, aumentando o escoamento superficial da água e a remoção das partículas do solo.

As culturas em sucessão de milho e feijão apresentaram uma perda média de solo de $0,10 \mathrm{Mg} \mathrm{ha}^{-1}$ ano ${ }^{1}$. Esses valores são reduzidos devido à aplicação da prática de plantio direto, que é caracterizada como uma técnica de conservação do solo que reduz a erosão hídrica pela permanência de culturas remanescentes, o que protege o solo e contribui com o aumento dos teores de matéria orgânica.

Nos povoamentos de eucalipto a perda de solo média foi de $3,77 \mathrm{Mg} \mathrm{ha}^{-1} \mathrm{ano}^{-1}$. Os valores estão acima da média da área, devido à ausência de manejos conservacionistas nessas áreas, caracterizados como plantio morro a baixo, que confere maior velocidade ao escoamento superficial das águas.

A mata nativa apresentou a menor média de perda de solo, calculada em $0,0087 \mathrm{Mg} \mathrm{ha}^{-1} \mathrm{ano}^{-1}$, com perda total de $0,60 \mathrm{Mg} a^{-1}{ }^{-1}$. No cultivo de café em nível, que corresponde a 54,31\% da área, a média das perdas de solo foi de 2,09 Mg ha ${ }^{-1}$ ano $^{-1}$ e a perda total de 497,85 $\mathrm{Mg}$ ano $^{-1}$. A contribuição das áreas de cafezais na perda total de solo é de $76,67 \%$ sendo, portanto, as áreas que mais fornecem sedimentos ao deflúvio.

\section{Tolerância de Perdas de Solo}

Os valores da TPS foram de 5,19, 5,69 e 5,90 $\mathrm{Mg} \mathrm{ha}^{-1}$ ano $^{-1}$, respectivamente, para os solos LVd1, LVd2 e LVd3. O resultado da variável h foi $1.000 \mathrm{~mm}$, o valor de ponderação da variável $\mathrm{r}_{\mathrm{a}}$ foi 1 e a ponderação das variáveis $\mathrm{m}$ e p resultou no valor 0,7 , com a permeabilidade do solo classificada como lenta. A densidade dos solos, em $\mathrm{g} \mathrm{cm}^{-3}$, foi de 1,060, 1,163 e 1,205, respectivamente, para os solos LVd1, LVd2 e LVd3. 


\section{Perdas de Solo nos Modelos Rusle E EPM}

Os valores das perdas de solo por unidade de área no modelo EPM foram transformados de $\mathrm{m}^{3} \mathrm{~km}^{-2}$ ano $^{-1}$ para $\mathrm{Mg} \mathrm{ha}^{-1}$ ano $^{-1}$, devido à utilização mais frequente da última no Brasil. Para conversão das unidades de volume para massa foi utilizado à densidade média do solo de $1,150 \mathrm{~g} \mathrm{~cm}^{-3}$. na Tabela 2

Os valores das perdas de solo nos modelos RUSLE (Figura 3A) e EPM (Figura 3B) estão descritos
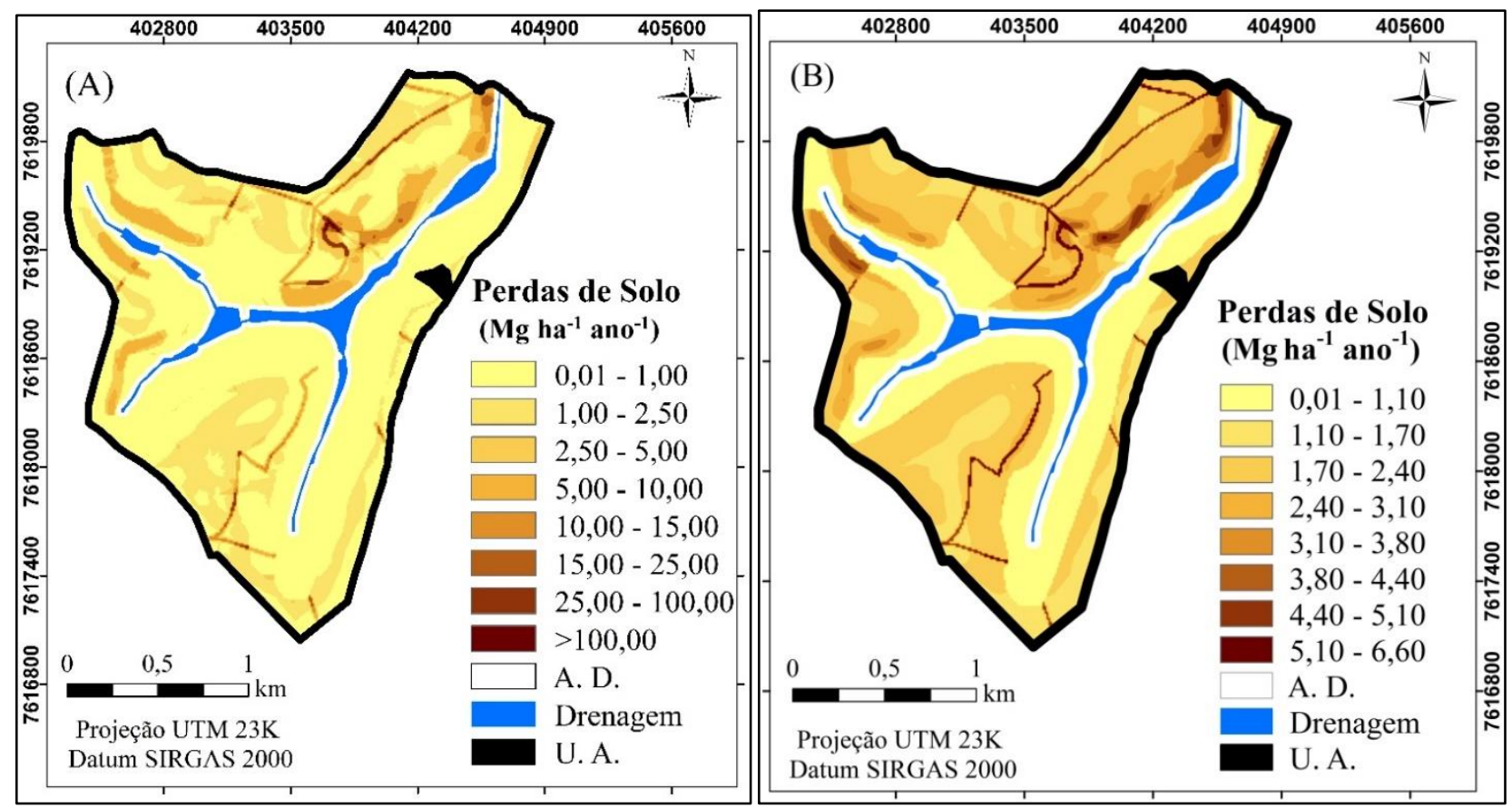

Figura 3: (A) Perdas de solo no modelo RUSLE com classificação adaptada de Beskow et al. (2009) e (B) Perdas de solo no modelo EPM com classificação de Gavrilovic (1988).

Tabela 2: Perdas médias de solo calculadas pelos modelos RUSLE e EPM.

\begin{tabular}{|c|c|c|c|c|c|c|c|}
\hline \multirow{3}{*}{$\begin{array}{l}\text { Classes de Uso } \\
\text { do Solo }\end{array}$} & \multirow{2}{*}{ Área } & \multicolumn{2}{|c|}{$\begin{array}{c}\text { Perda média de } \\
\text { solo }\end{array}$} & \multicolumn{2}{|c|}{ Perda total de solo } & \multicolumn{2}{|c|}{$\begin{array}{l}\text { Contribuição nas } \\
\text { perdas de solo }(\%)\end{array}$} \\
\hline & & RUSLE & EPM & RUSLE & EPM & & \\
\hline & ha & \multicolumn{2}{|c|}{$\mathrm{Mg} \mathrm{ha}^{-1} \mathrm{ano}^{-1}$} & \multicolumn{2}{|c|}{$\mathrm{Mg}$ ano $^{-1}$} & \multicolumn{2}{|c|}{ RUSLE $\quad$ EPM } \\
\hline Café & 237,35 & 1,52 & 2,0900 & 360,75 & 497,85 & 56,12 & 76,670 \\
\hline Milho + Feijão & 60,49 & 0,04 & 0,1000 & 2,42 & 33,42 & 1,08 & 5,140 \\
\hline Eucalipto & 16,95 & 6,05 & 3,7700 & 102,42 & 63,98 & 15,42 & 9,850 \\
\hline Mata Nativa & 68,99 & 0,05 & 0,0087 & 2,47 & 0,60 & 0,10 & 0,092 \\
\hline Solo exposto & 9,71 & 18,64 & 5,5000 & 180,81 & 53,46 & 27,28 & 8,230 \\
\hline Usina de adubo & 2,40 & - & - & - & - & - & - \\
\hline Drenagem & 19,51 & - & - & - & - & - & - \\
\hline $\begin{array}{l}\text { Área de } \\
\text { deposição }\end{array}$ & 21,60 & - & - & - & - & - & - \\
\hline Total & 437 & $1,57^{*}$ & $1,46^{*}$ & 648,87 & 649,31 & 100 & 100 \\
\hline
\end{tabular}

Notas: RUSLE: Equação Universal da Perda de Solo Revisada. EPM: Método de Erosão Potencial.

A distribuição das perdas de solo na sub-bacia, em ambos os modelos, apresenta essencialmente áreas com erosão suave. Todavia, expõe áreas prioritárias para a implantação de manejos conservacionistas que amenizem os efeitos deletérios da erosão. No modelo RUSLE cerca de 7\% da área possui perdas de solo acima do limite da TPS, localizadas nos povoamentos de eucalipto e nos carreadores com solo exposto. No modelo EPM, tais áreas apresentam uma taxa de erosão máxima de $5,50 \mathrm{Mg} \mathrm{ha}^{-1}$ ano $^{-1}$, ligeiramente abaixo do limite da TPS. Logo, as mesmas devem ser objeto de monitoramento e mitigação das taxas de erosão. 


\section{CONCLUSÕES}

As estimativas de perdas de solo no modelo EPM variaram entre 0,0087 e 5,50 $\mathrm{Mg} \mathrm{ha}^{-1} \mathrm{ano}^{-1}$ e no modelo RUSLE entre 0,05 e $18,64 \mathrm{Mg} \mathrm{ha}^{-1}$ ano $^{-1}$.

A perda média de solo no modelo EPM foi de 1,46 $\mathrm{Mg} \mathrm{ha}^{-1}$ ano $^{-1}$ e no modelo RUSLE de 1,57 Mg ha${ }^{1}$ ano $^{-1}$, a baixo do limite mínimo da TPS, que variou entre 5,19 e 5,90 $\mathrm{Mg} \mathrm{ha}^{-1}$ ano $^{-1}$.

No modelo RUSLE as áreas com cultivo de eucalipto possuem perdas de solo de $6,05 \mathrm{Mg} \mathrm{ha}^{-1} \mathrm{ano}^{-1} \mathrm{e}$ os carreadores de café de $18,64 \mathrm{Mg} \mathrm{ha}^{-1}$ ano $^{-1}$, já no modelo EPM tais perdas são, respectivamente, 3,77 e 5,50 $\mathrm{Mg} \mathrm{ha}^{-1} \mathrm{ano}^{-1}$.

Nos dois modelos estudados, as maiores perdas de solo estão associadas aos povoamentos de eucalipto e solos expostos. Todavia, apesar do modelo EPM não apresentar área com taxa de erosão acima do limite da TPS, ambos os modelos indicam tais áreas como prioritárias para a mitigação das taxas de erosão.

\section{REFERÊNCIAS}

AQUINO, R. F.; MITAS, L.; BROWN, W. M.; JOHNSTON, D. M. Spatial variability of the rainfall erosivity in Southern region of Minas Gerais State, Brazil. Ciência e Agrotecnologia, Lavras, v. 36, n. 5, p. 533-542, set. /out.2012.

AYER, J. E. B.; OLIVETTI, D.; MINCATO, R. L.; SILVA, M. L. N. Erosão hídrica em Latossolos Vermelhos Distróficos. Pesquisa Agropecuária Tropical, Goiânia, v. 45, n. 2, p. 180-191, abr./jun. 2015.

BERTOL, I.; ALMEIDA, J. A. Tolerância de perda de solo por erosão para os principais solos do estado de Santa Catarina. Revista Brasileira de Ciência do Solo, Viçosa, v. 24, n. 3, p. 657-668, mai. 2000.

BERTONI, J.; LOMBARDI NETO, F. Conservação do solo. São Paulo: 8 Ed. Ícone, 2012. 360p

BESKOW, S.; MELLO, C. R.; NORTON, L. D.; CURI, N.; VIOLA, M. R.; AVANZI, J. C. Soil erosion prediction in the Grande River Basin, Brazil using distributed modeling. Catena, Amsterdam, v. 79, n. 1, p. 49-59, oct. 2009.

BRASIL. Código Florestal (2012). Lei $\mathbf{n}^{\circ} \mathbf{1 2 . 6 5 1}$ de 25 de maio de 2012. Brasília, DF: Congresso Nacional, 2012.

BRASIL. Ministério da Agricultura. (1962). Levantamento de reconhecimento dos solos da região sob influência do reservatório de Furnas. Rio de Janeiro, RJ: Serviço Nacional de Conservação dos Solos, Boletim Técnico n. 13, 1962.

CÂNDIDO, B. M.; SILVA, M. L. N.; CURI, N.; BATISTA, P. V. G. Erosão Hídrica Pós-Plantio em Florestas de Eucalipto na Bacia do Rio Paraná, no Leste do Mato Grosso do Sul. Revista Brasileira de Ciência do Solo, Viçosa, v. 38, n. 5, p. 1565-1575, set./out. 2014.

CARVALHO R.; SILVA, M. L. N.; AVANZI, J. C.; CURI, N.; SOUZA, F. S. Erosão Hídrica em Latossolo Vermelho sob Diversos Sistemas de Manejo do Cafeeiro no Sul de Minas Gerais. Ciência e Agrotecnologia. Lavras, v. 31, n. 6, p. 1679-1687, nov./dec. 2007.

EMBRAPA, Empresa Brasileira de Pesquisa Agropecuária. Centro Nacional de Pesquisas de Solos. Manual de métodos de análise de solos. 2 ed. Rio de Janeiro, 2011.

EMBRAPA, Empresa Brasileira de Pesquisa Agropecuária - EMBRAPA. Centro Nacional de Pesquisas de Solos. Sistema Brasileiro de Classificação de Solos. 3 ed. Rio de Janeiro, 2013.

GALINDO, I. C. L.; MARGOLIS, E. Tolerância de perdas por erosão para solos do Estado de Pernambuco. Revista Brasileira de Ciência do Solo, Viçosa, v. 13, n. 1, p. 95-100, out. 1989.

GAVRILOVIC, Z. The use of an Empirical Method (Erosion Potential Method) for Calculating Sediment production and Transportation in Unstudied or Torrential Streams. In: WHITE, W. R. (ed.). International Conference on River Regime Hydraulics Research Limited; p. 18-20, mai. 1988; Oxon UK. Wallingford: Hydraulics Research Limited; 1988. p. 411-422.

HASUI, Y. A grande colisão pré-cambriana do Sudeste Brasileiro e a estruturação regional. Geociências, Rio Claro, v. 29, n. 2, p. 141-169, set. 2010. 
IBGE, Instituto Brasileiro de Geografia e Estatística. Carta Topográfica do Município de Alfenas (FOLHA SF 23-1-1-3). 1 ed. Rio de Janeiro, 1970.

IBGE, Instituto Brasileiro de Geografia e Estatística. Manual técnico de pedologia. 3 ed. Rio de Janeiro, 2015.

MCBRATNEY, A. B.; MENDONÇA, S. M. L.; MINASNY B. On digital soil mapping. Geoderma, Beijing, v. 117, n. 1-2, p. 3-52, jun. 2003.

MITASOVA, H. M.; MITAS, L.; BROWN, W. M.; JOHNSTON, D. M. Terrain modelling and soil erosion: applications for Ft. Hood report for USA CERL. Net, Champaign, University of Illinois, 1996. Disponível em: <http://shagit.meas.ncsu.edu/

helena/gmslab/reports/cer101 /finalreport/report01/default.htm>. Acesso em: 17 mai. 2016.

OLIVETTI, D.; MINCATO, R. L.; AYER, J. E. B.; SILVA, M. L. N.; CURI, N. Spatial and Temporal Modeling of Water Erosion in Dystrophic Red Latosol (Oxisol) used for Farming and Cattle Raising Activities in a Sub-Basin in the South of Minas Gerais. Ciência e Agrotecnologia, Lavras, v. 39, n. 1, p. 586, jan./feb. 2015.

PANAGOS, P.; BORRELLI, P.; MEUSBURGER, K. A New European Slope Length and Steepness Factor (LS-Factor) for Modeling Soil Erosion by Water. Geosciences, Basel, v. 5, n. 2, p. 117-126, mar./apr. 2015.

PIMENTEL, D.; HARVEY, C.; RESOSUDARMO, P.; SINCLAIR, K.; KURZ, D.; MCNAIR, M.; CRIST, S.; SHPRITZ, L.; FITTON, L.; SAFFOURI, R.; BLAIR, R. Environmental and economic costs of soil erosion and conservation benefits. Science, Washington, v. 267, n. 5201, p. 1117-1123, mar. 1995.

RENARD, K. G.; FOSTER, G. R.; WEESIES, G. A.; MCCOOL, D. K.; YODER, D. C. Predicting Soil Erosion by Water: A Guide to Conservation Planning with the Revised Universal Soil Loss Equation (RUSLE). U.S. Department of Agriculture, Agriculture Handbook, v.703, 1997. 385p

ROOSE, E. J. Application of the Universal Soil Loss Equation of Wischmeier and Smith in West Africa. In: Greenland DJ, Lal R. (eds.) Soil conservation and management in the humid tropics. 1 ed. Chichester: John Wiley \& Sons. 1977, p. 177-187

SANTOS, R. D.; LEMOS, R. C.; SANTOS, H. G.; KER, J. C.; ANJOS, L. H. C. Manual de descrição e coleta de solos no campo.5th ed. Viçosa, Sociedade Brasileira de Ciência do Solo - SBCS, Empresa Brasileira de Pesquisa Agropecuária - Embrapa, Centro Nacional de Pesquisa de Solos - CNPS, 2005.

SILVA, B. P. C.; SILVA, M. L. N.; BATISTA, P. V. G.; PONTES, L. M.; ARAÚJO, E. F.; CURI, N. Soil and water losses in eucalyptus plantation and natural forest and determination of the USLE factors at a pilot sub-basin in Rio Grande do Sul, Brazil. Ciência \& Agrotecnologia, Lavras, v. 40, n. 4, p. 432-442, Jul./Aug. 2016

SILVA, M. L. N.; CURI, N.; FERREIRA, M. M.; LIMA, J. M.; FERREIRA, D. F. Proposição Proposição de modelos para estimativa da erodibilidade de Latossolos brasileiros. Pesquisa Agropecuária Brasileira, Brasília, v. 34, n. 12, p. 2287-2298, dez.1999.

SILVA, S.A.; LIMA, J. S. S.; OLIVEIRA, R. B.; SOUZA, G. S.; SILVA, M. A. Análise espacial da erosão hídrica em um latossolo vermelho amarelo sob cultivo de café conilon. Revista Ciência Agronômica, Fortaleza, v. 38, n. 4, p. 335-342, out. 2007.

SOIL SURVEY DIVISION STAFF. Soil survey manual. Soil Conservation Service. Handbook 18, Washington, D.C.: U.S. Department of Agriculture, 1993.

SPALEVIC, V. Impact of land use on runoff and soil erosion in Polimlje. Doctoral thesis, Faculty of Agriculture of the University of Belgrade, Serbia, 260p. 2011.

SPAROVEK, G.; VAN LIER, Q. J.; DOURADO NETO, D. Computer assisted Köppen climate classification: a case study for Brazil. International Journal of Climatology, London, v. 27, n. 2, p. 257-266, oct. 2007.

WISCHMEIER, W. H.; SMITH, D. D. Predicting rainfall erosion losses a guide to conservation planning. Agriculture Handbook 537, Washington, D.C.: U.S. Department of Agriculture, 1978. 58p.

ZHANG, R. Determination of soil sorptivity and hydraulic conductivity from the disk infiltrometer. Soil Science Society of America Journal, Madison, v. 61, n. 2, p. 1024-1030, may/aug. 1997. 